\title{
Fast and Accurate Mode-Spectrum Analysis of Waveguide Structures Using a New Set of Edge-Conditioned Basis Functions
}

\author{
John M. Damaschke, Smain Amari, Jens Bornemann \\ Laboratory for Lightwave Electronics, Microwaves and Communications, Department of Electrical and \\ Computer Engineering, University of Victoria, Victoria, British Columbia, Canada V8W 3P6
}

Received 10 January 1997; revised 22 July 1997

\begin{abstract}
A new set of edge-conditioned basis functions is used to determine the modal characteristics of a large variety of nonstandard waveguide structures. Since the electromagnetic field includes the proper edge conditions at metallic corners and since the new basis functions are readily Fourier transformed, a considerable reduction in computational effort with respect to standard modal field-matching techniques is observed. Similar to procedures involving other known sets of applicable basis functions, the analysis achieves a higher reliability level, as it completely avoids relative convergence phenomena.

The method is demonstrated at the examples of several waveguide structures, such as the L-shape, T-shape, and cross-iris-shape waveguides; the rectangular coaxial waveguide; and the ridged circular waveguide. Results for the cut-off wave numbers of TE and TM modes are shown to be in excellent agreement with those from standard modal field-matching techniques and previously published data. (c) 1998 John Wiley \& Sons, Inc. Int J RF and Microwave CAE 8: 215-225, 1998.
\end{abstract}

Keywords: integral-equation technique; basis functions; mode-matching technique; eigenvalues/eigenfunctions

\section{INTRODUCTION}

Modern integrated waveguide technology employs nonstandard waveguide cross sections to take advantage of such features as enlargement of monomode bandwidth, field concentration in specific regions of the guide, field rotation, excitation of individual modes, and cross-coupling in dual-mode arrangements, to name only a few. Many of those structures are formed by permuting standard rectangular or circular waveguides in certain regions, thus resulting in L-shape, T-shape, cross-iris, or ridged configurations, e.g., [1-4, 6, 8, 9]. They are typically used in dual-mode applications, e.g., as in waveguide filters, polarizers, or orthomode transducers. For the accurate analysis

Correspondence to: Prof. Jens Bornemann and design of such components, the knowledge of the eigenmode spectrum is of fundamental importance, as it determines such critical quantities as resonator lengths, intercavity direct or crosscoupling, phase relationships, etc. Moreover, the determination of the mode spectrum is the most time-consuming and most critical part of a threedimensional modal analysis [10].

Several publications have been concerned with this topic [1-3]. Typically, modal field-matching techniques are applied, as they offer a versatile tool for analyzing this kind of structure [8-10]. Although good results can be achieved in general, there are several shortcomings. The fields along the interface between different subregions of the cross section (e.g., Fig. 1) are expressed in terms of pure sine and cosine functions. Therefore, they usually do not satisfy the boundary condition for 


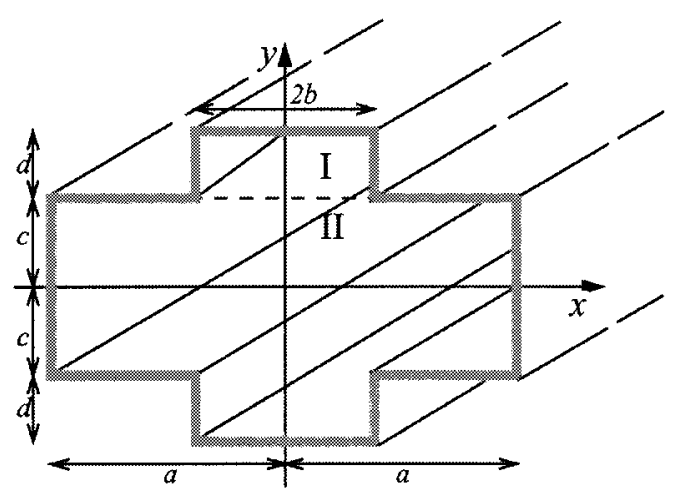

a)

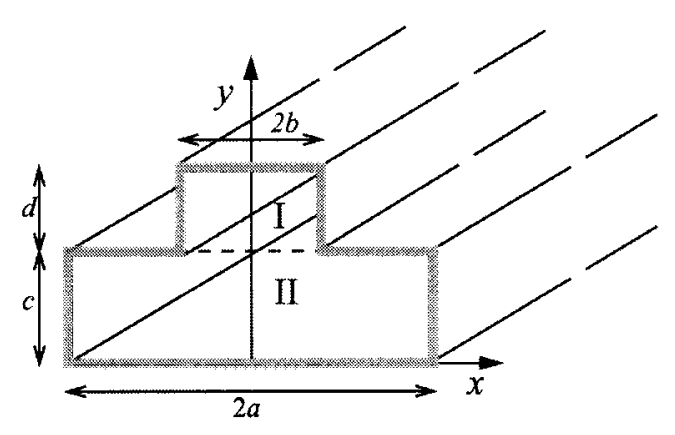

c)

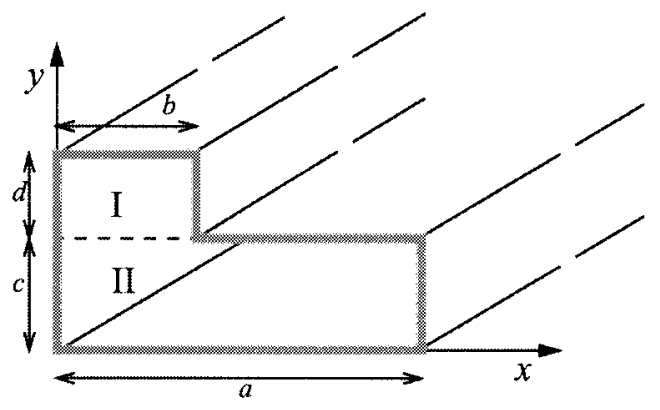

b)

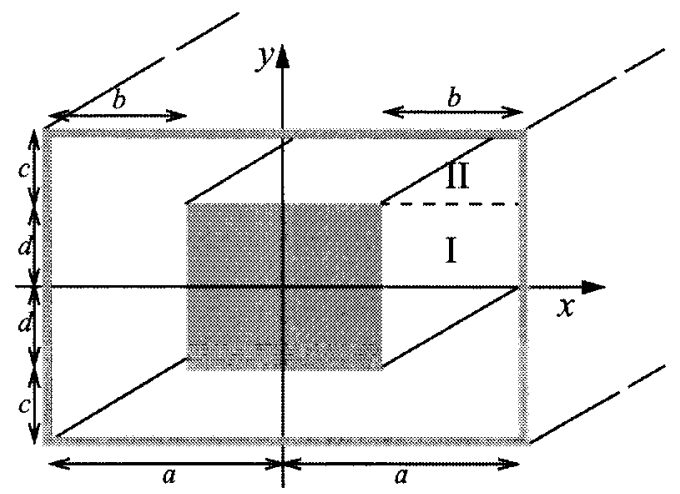

d)

Figure 1. Geometry of analyzed rectangular waveguides: (a) Cross-iris; (b) L-shaped; (c) T-shaped; (d) rectangular coaxial.

the larger region, or the edge condition at a metal corner, i.e., the singularity of the fields. To achieve a satisfactory approximation of the field at the interface and thus to get reliable results, a large number of modes is usually required, particularly if the electromagnetic field itself is to be computed. In addition, we encounter the problem of relative convergence, which is especially important for large dimensional differences between subregions.

To overcome these deficiencies, the fields at discontinuities are expanded in terms of edgeconditioned basis functions (as is well known from, e.g., the spectral-domain approach [5], and the resulting system of equations is solved using the method of moments [11]. Several attempts have been made to apply this method to waveguidebased discontinuities. In [12], weighted Chebyshev polynomials are used as basis functions for zerothickness diaphragms, whereas weighted Geigenbauer polynomials describe the fields in finitethickness applications (e.g., [13-15]).
This paper focuses on a new set of basis functions upon which three distinct criteria have been imposed. First, to obtain accurate results, the basis functions must satisfy the edge conditions of the respective field components at $90^{\circ}$ metallic corners. Second, the Fourier sine and Fourier cosine integrals of such basis functions must be solvable analytically, which contributes to both the speed and accuracy of the solution, as the Fourier spectra can be calculated without any need for numerical integration. Third, the sinusoidal dependence of the regular waveguide modes shall be reflected in the basis functions to maintain an appropriate transition of the fields at the discontinuity to those away from the discontinuity, the latter of which remain, of course, a superposition of sinusoidal waveguide-mode functions.

Consequently, the resulting set of basis functions can be used for a large variety of different geometries which, first, involve $90^{\circ}$ metallic corners and, second, require the respective field 
component to be expressed in terms of sine or cosine functions, independent of whether the waveguide housing is of rectangular or circular shape.

The theory is verified by comparing our results with previously published data, and with the standard mode-matching technique. It is demonstrated that two basis functions are sufficient, not only to determine the complete mode spectrum of the waveguides, but also to accurately represent the fields at or in the immediate vicinity of the discontinuity, which, in standard mode-matching procedures, requires an extremely large number of modes.

\section{THEORY}

\section{A. Cross-Iris Waveguide}

First we will consider the rectangular structures shown in Fig. 1, of which the cross iris (Fig. 1a) is the most general case. Field symmetry is assumed with respect to the planes $x=0$ and $y=0$. Two electric walls, one at $x=0$ and one at $y=0$, lead to the L-shaped waveguide of Fig. 1b, and one magnetic and one electric wall yield the additional modes in a T-shaped waveguide (Fig. 1c). For the complete modal analysis of the cross-iris (Fig. 1a), the additional case of two magnetic walls is investigated. The rectangular coaxial waveguide (Fig. 1d) is presented in the next section (Rectangular Coaxial Waveguide).

For the analysis, the axial fields inside the waveguide are written in terms of two-dimensional Fourier series for the two regions, I and II (e.g., $[5,6])$. In the following, the expressions are given for TE modes with electric wall symmetry at $y=0$ and magnetic wall symmetry at $x=0$. The boundary conditions are included in the expansions, whereas, so far, no conditions are imposed at the interface. In this way, the magnetic fields $H_{z}$ are obtained as

$$
\begin{aligned}
H_{z}^{\mathrm{I}}= & \sum_{m=1}^{\infty} A_{m}^{\mathrm{I}} \cdot \sin \frac{(2 m-1) \pi x}{2 b} \\
& \cdot \cosh \left(\gamma_{1 m}(y-d-c)\right)
\end{aligned}
$$

in region $\mathrm{I}$, i.e., $0 \leq x \leq b, c<y \leq c+d$, and

$$
H_{z}^{\mathrm{II}}=\sum_{m=1}^{\infty} A_{m}^{\mathrm{II}} \cdot \sin \frac{(2 m-1) \pi x}{2 a} \cdot \cosh \left(\gamma_{2 m} y\right)
$$

in region $\mathrm{II}$, i.e., $0 \leq x \leq a, 0 \leq y \leq c$, respectively, where

$$
\gamma_{1 m}=\sqrt{\left(\frac{2 m-1}{2 b} \pi\right)^{2}-k_{c}^{2}}
$$

and

$$
\gamma_{2 m}=\sqrt{\left(\frac{2 m-1}{2 a} \pi\right)^{2}-k_{c}^{2}}
$$

$k_{c}$ is the free-space wave number. The electric fields in the two regions are given by $E_{x} \sim$ $\partial H_{z} / \partial y$.

Now the tangential electric field at the interface plane, $y=c$, is expressed in terms of basis functions:

$$
\begin{aligned}
E_{x} & =\sum_{n=1}^{N} c_{n} \cdot B_{n}(x) \\
& =\sum_{n=1}^{N} c_{n} \cdot \frac{\sin ((2 n-1) \pi x /(2 b))}{\left(1-(x / b)^{2}\right)^{1 / 3}}
\end{aligned}
$$

where $c_{n}$ are the unknown expansion coefficients. Note that this expression satisfies both the boundary conditions of the electric field at the interface as well as the edge condition. In particular, it becomes singular as $(b-x)^{-1 / 3}$ in the vicinity of the metal edge, which resembles the characteristic behavior of the electric field. This reduces the required number of basis functions and increases the efficiency of the algorithm. The next step is to write the Fourier coefficients, $A_{m}^{\mathrm{I}}$ and $A_{m}^{\mathrm{II}}$, in terms of the expansion coefficients, $c_{n}$. This is done by matching the Fourier sine or cosine integrals of the tangential electric fields in the two different regions, $E_{x}^{\mathrm{I}}$ and $E_{x}^{\mathrm{II}}$. For the assumed symmetry this yields

$$
\begin{gathered}
\int_{0}^{b} E_{x}^{\mathrm{I}}(x, y=c) \cdot \sin \frac{(2 m-1) \pi x}{2 b} d x \\
=\int_{0}^{b} E_{x} \cdot \sin \frac{(2 m-1) \pi x}{2 b} d x \\
\int_{0}^{a} E_{x}^{\mathrm{II}}(x, y=c) \cdot \sin \frac{(2 m-1) \pi x}{2 a} d x \\
=\int_{0}^{b} E_{x} \cdot \sin \frac{(2 m-1) \pi x}{2 a} d x
\end{gathered}
$$


where $E_{x}$ is as given in (3). These integrals can be solved analytically and lead to a sum of Bessel functions [7]. From this, the Fourier coefficients are expressed as

$$
\begin{aligned}
& A_{m}^{\mathrm{I}}=\frac{2}{b} \cdot \frac{\sum_{n=1} c_{n} \cdot \tilde{B}_{n}^{\mathrm{I}}(m)}{\gamma_{1 m} \cdot \sinh \left(\gamma_{1 m} d\right)} \\
& A_{m}^{\mathrm{II}}=-\frac{2}{a} \cdot \frac{\sum_{n=1} c_{n} \cdot \tilde{B}_{n}^{\mathrm{II}}(m)}{\gamma_{2 m} \cdot \sinh \left(\gamma_{2 m} c\right)}
\end{aligned}
$$

where the $\tilde{B}_{n}^{\mathrm{I}}(m)$ and $\tilde{B}_{n}^{\mathrm{II}}(m)$ denote the spectra of the basis functions:

$$
\begin{aligned}
\tilde{B}_{n}^{\mathrm{I}}(m)= & \int_{0}^{b} \frac{\sin ((2 n-1) \pi x /(2 b))}{\left(1-(x / b)^{2}\right)^{1 / 3}} \\
& \cdot \sin \frac{(2 m-1) \pi x}{2 b} d x \\
= & \frac{b}{4} \Gamma\left(\frac{2}{3}\right) \sqrt{\pi}\left(-\frac{J_{1 / 6}|\pi(n+m-1)|}{|\pi / 2 \cdot(n+m-1)|^{1 / 6}}\right. \\
& \left.+\frac{J_{1 / 6}|\pi(n-m)|}{|\pi / 2 \cdot(n-m)|^{1 / 6}}\right)
\end{aligned}
$$

$\tilde{B}_{n}^{\mathrm{II}}(m)$

$$
\begin{aligned}
= & \int_{0}^{b} \frac{\sin ((2 n-1) \pi x /(2 b))}{\left(1-(x / b)^{2}\right)^{1 / 3}} \\
& \cdot \sin \frac{(2 m-1) \pi x}{2 a} d x \\
= & \frac{b}{4} \Gamma(2 / 3) \sqrt{\pi} \\
& \cdot\left(-\frac{J_{1 / 6}(\pi / 2)((2 n-1)+(2 m-1)(b / a)) \mid}{|(\pi / 4)((2 n-1)+(2 m-1)(b / a))|^{1 / 6}}\right. \\
& \left.+\frac{J_{1 / 6}|(\pi / 2)((2 n-1)-(2 m-1)(b / a))|}{|(\pi / 4)((2 n-1)-(2 m-1)(b / a))|^{1 / 6}}\right)
\end{aligned}
$$

Now all field components depend only on the $c_{n}$ 's. Rewriting the continuity condition of $H_{z}$ at the interface plane in terms of the expansion coefficients and applying Galerkin's method yields a set of linear equations in $c_{n}$,

$$
[G][c]=0
$$

where the elements of the matrix $[G]$ are given by

$$
\begin{array}{r}
g_{i j}=\sum_{m=1}^{\infty}\left(\frac{\gamma_{1 m}}{b \cdot \tanh \left(\gamma_{1 m} d\right)} \tilde{B}_{j}^{\mathrm{I}}(m) \tilde{B}_{i}^{\mathrm{I}}(m)\right. \\
\left.+\frac{\gamma_{2 m}}{a \cdot \tanh \left(\gamma_{2 m} c\right)} \tilde{B}_{j}^{\mathrm{II}}(m) \tilde{B}_{i}^{\mathrm{II}}(m)\right) \\
i, j=1, \ldots, N
\end{array}
$$

The roots of the determinant of $[G]$ represent the results for the cut-off wave numbers. Finding the roots, however, can be simplified by looking at the smallest singular value [9], since the poles in the determinant are avoided. Note that the sum in (11) is taken over the modal index $m$ and that the actual modal distributions in regions I and II are only present in the spectra $\tilde{B}_{j, i}^{\mathrm{I}, \mathrm{II}}(\mathrm{m})$ of the basis functions and not (as in standard field-matching techniques) as separate entries in the system matrix. Therefore, the matrix elements $g_{i j}$ in (11) can be tested for convergence prior to calculating the singular value, and hence the phenomenon of relative convergence is avoided.

The calculations for the other symmetry conditions are obtained similarly. The Fourier expansions and basis functions are changed according to the different boundary conditions, leading to very similar expressions. Furthermore, the results for TM modes are analogous. Here the axial electric field is written in terms of a Fourier series, and, again, the transverse electric fields in the two regions are matched at the interface, which allows the Fourier coefficients to be expressed in terms of the expansion coefficients. The continuity condition of the magnetic fields $H_{x}$ is then used to derive the integral equation, and Galerkin's method is used to find the cut-off wave numbers.

\section{B. Rectangular Coaxial Waveguide}

The same approach is used to analyze the rectangular coaxial waveguide in Fig. 1d. For TE modes and magnetic wall symmetry at the $x=0$ and $y=0$ planes, the axial magnetic fields are expanded as

$$
\begin{aligned}
H_{z}^{\mathrm{I}}= & \sum_{m=1}^{\infty} A_{m}^{\mathrm{I}} \cdot \cos \frac{(m-1) \pi(a-x)}{b} \\
& \cdot \sinh \left(\gamma_{1 m} y\right)
\end{aligned}
$$




$$
\begin{aligned}
H_{z}^{\mathrm{II}}= & \sum_{m=1}^{\infty} A_{m}^{\mathrm{II}} \cdot \cos \frac{(2 m-1) \pi(a-x)}{2 a} \\
& \cdot \cosh \left(\gamma_{2 m}(y-d-c)\right)
\end{aligned}
$$

in region $\mathrm{I}$, i.e., $a-b \leq x \leq a, 0 \leq y \leq d$, and region II, i.e., $0 \leq x \leq a, d<y \leq c+d$, respectively, where

$$
\begin{aligned}
& \gamma_{1 m}=\sqrt{\left(\frac{m-1}{b} \pi\right)^{2}-k_{c}^{2}}, \\
& \gamma_{2 m}=\sqrt{\left(\frac{2 m-1}{2 a} \pi\right)^{2}-k_{c}^{2}}
\end{aligned}
$$

and $k_{c}$ is the free-space wave number. The basis functions

$$
B_{n}(x)=\frac{\cos ((n-1) \pi(a-x) / b)}{\left(1-((a-x) / b)^{2}\right)^{1 / 3}}
$$

are chosen independently from the symmetry conditions since, for this geometry, the symmetry axes do not affect the boundary conditions at the interface. (Note that the properties of the fundamental TEM mode on this structure are well known and need not be included in the determination of the mode spectrum.) Again, after applying Galerkin's method, the singular value decomposition is used to locate the roots in the system determinant function. As a by-product, the solution for the expansion coefficients is obtained that directly determines the tangential electric field at the interface as presented in the Results.

\section{Circular Ridged Waveguide}

The circular ridged waveguide in Figure 2 is solved in cylindrical coordinates. Although the field components involve Bessel functions, the angular dependence is given by sines and cosines, and therefore the previously used set of basis functions can be applied here. For TE modes and magnetic wall symmetry, the axial magnetic field is expanded as

$$
H_{z}^{\mathrm{I}}=\sum_{m=1}^{\infty} A_{m}^{\mathrm{I}} \cdot J_{m}\left(k_{c} \rho\right) \cdot \sin (m \phi)
$$

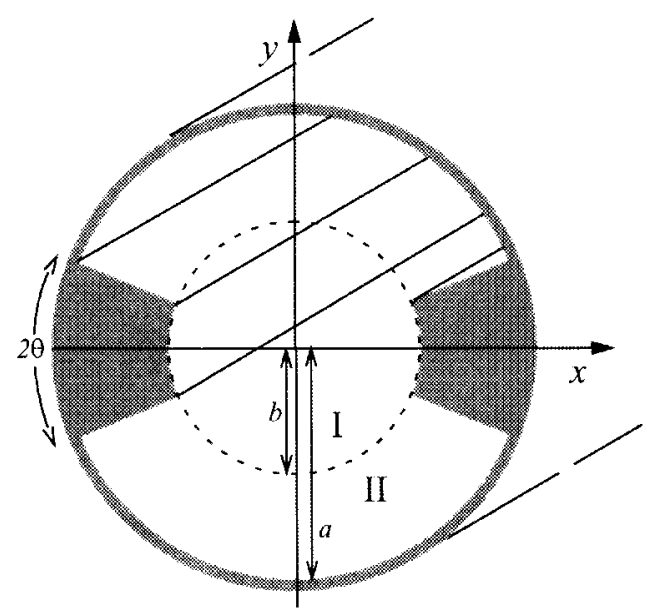

Figure 2. Geometry of ridged circular waveguide.

$$
\begin{aligned}
H_{z}^{\mathrm{II}}= & \sum_{m=1}^{\infty} D_{m}^{\mathrm{II}}\left[J_{l}^{\prime}\left(k_{c} a\right) Y_{l}\left(k_{c} \rho\right)\right. \\
& \left.-Y_{l}^{\prime}\left(k_{c} a\right) J_{l}\left(k_{c} \rho\right)\right] \cos \left(\frac{m \pi \phi}{2(\pi-\theta)}\right)
\end{aligned}
$$

in the two subregions, respectively. The electric fields are determined from this by $E_{\phi} \sim \partial H_{z} / \partial \rho$. The basis functions are chosen as

$$
B_{m}(\phi)=\frac{\cos (m \pi(\phi-\theta) /(\pi-2 \theta))}{((\phi-\theta)(\pi-\theta-\phi))^{1 / 3}}
$$

with their spectra

$$
\begin{aligned}
\tilde{B}_{n}^{\mathrm{I}}(m)= & \frac{2}{\pi} \cdot \int_{\theta}^{(\pi-\theta)} \sin (m \phi) \\
& \times \frac{\cos (m \pi(\phi-\theta) /(\pi-2 \theta))}{((\phi-\theta)(\pi-\theta-\phi))^{1 / 3}} d \phi \\
= & \frac{1}{2 \pi} \Gamma\left(\frac{2}{3}\right) \sqrt{\pi} \cdot\left(\sin \frac{m+n}{2} \pi\right. \\
& \cdot \frac{J_{1 / 6}|((m+n) / 2) \pi-m \theta|}{|((m+n) / 4) \pi-m \theta / 2|^{1 / 6}} \\
& +\sin \frac{m-n}{2} \pi \\
& \left.\cdot \frac{J_{1 / 6}|((m-n) / 2) \pi-m \theta|}{|((m-n) / 4) \pi-m \theta / 2|^{1 / 6}}\right)
\end{aligned}
$$




$$
\begin{aligned}
\tilde{B}_{n}^{\mathrm{II}}(m)= & \frac{2}{\pi-2 \theta} \cdot \int_{\theta}^{(\pi-\theta)} \cos \left(\frac{n \pi(\phi-\theta)}{\pi-2 \theta}\right) \\
& \times \frac{\cos (m \pi(\phi-\theta) /(\pi-2 \theta))}{((\phi-\theta)(\pi-\theta-\phi))^{1 / 3}} d \phi \\
= & \frac{1}{\pi-2 \theta} \frac{\Gamma(2 / 3)}{2} \frac{\sqrt{\pi}}{1+\delta_{m 0}} \\
& \times\left(\cos \frac{m-n}{2} \pi \cdot \frac{J_{1 / 6}|((m-n) / 2) \pi|}{|((m-n) / 4) \pi|^{1 / 6}}\right. \\
& \left.+\cos \frac{m+n}{2} \pi \cdot \frac{J_{1 / 6}|((m+n) / 2) \pi|}{|((m+n) / 4) \pi|^{1 / 6}}\right)
\end{aligned}
$$

Again, the solution is given by a linear matrix equation,

$$
[G][c]=0
$$

where the elements of the matrix $[G]$ are given by

$$
\begin{aligned}
g_{i j}= & \frac{1-\theta}{\pi} \cdot \sum_{m=0}^{\infty}\left(\left(1+\delta_{m 0}\right) \tilde{B}_{j}^{\mathrm{II}}(m) \tilde{B}_{i}^{\mathrm{II}}(m)\right. \\
& \left.\cdot \frac{Y_{l}^{\prime}\left(k_{c} a\right) J_{l}\left(k_{c} b\right)-J_{l}^{\prime}\left(k_{c} a\right) Y_{l}\left(k_{c} b\right)}{Y_{l}^{\prime}\left(k_{c} a\right) J_{l}^{\prime}\left(k_{c} b\right)-J_{l}^{\prime}\left(k_{c} a\right) Y_{l}^{\prime}\left(k_{c} b\right)}\right) \\
& -\sum_{n=0}^{\infty}\left(\frac { J _ { n } ( k _ { c } b ) } { J _ { n } ^ { \prime } ( k _ { c } b ) } \left[B_{i}^{\mathrm{I}}(n) B_{j}^{\mathrm{I}}(n)\left(1+\delta_{m 0}\right)\right.\right. \\
& \left.\left.+B_{i}^{\mathrm{I}}(n) B_{j}^{\mathrm{I}}(n)\right]\right) \quad i, j=1, \ldots, N \quad(21)
\end{aligned}
$$

For implementation of the algorithm in a computer code, it should be noted that the Fourier spectra of the basis functions in (8), (9), and (18), (19) are frequency independent and, consequently, are computed only once to speed up the calculations considerably. (A detailed presentation of the computation of fractional-order Bessel functions is given in [15] along with a ready-toimplement Fortran code. The reader is referred to this reference for further details.) Since the Bessel routine of [15] is extremely fast, the computation of the frequency-independent Fourier spectra is negligible compared to the subsequent search algorithm for solving (10) or (20).

\section{RESULTS}

Figure 3 shows the results for the L-shaped waveguide of Fig. 1b. Excellent agreement with [2] is observed for both TE modes and TM modes. Note that, because of the symmetric L-shape, the normalized cut-off frequencies of modes $i k$ are identical to those of modes $\mathrm{ki}$. Figure 4 depicts the results for TE modes of the T-shaped waveguide of Fig. 1c, and the results are practically identical to those presented in [1]. Figure 5 shows

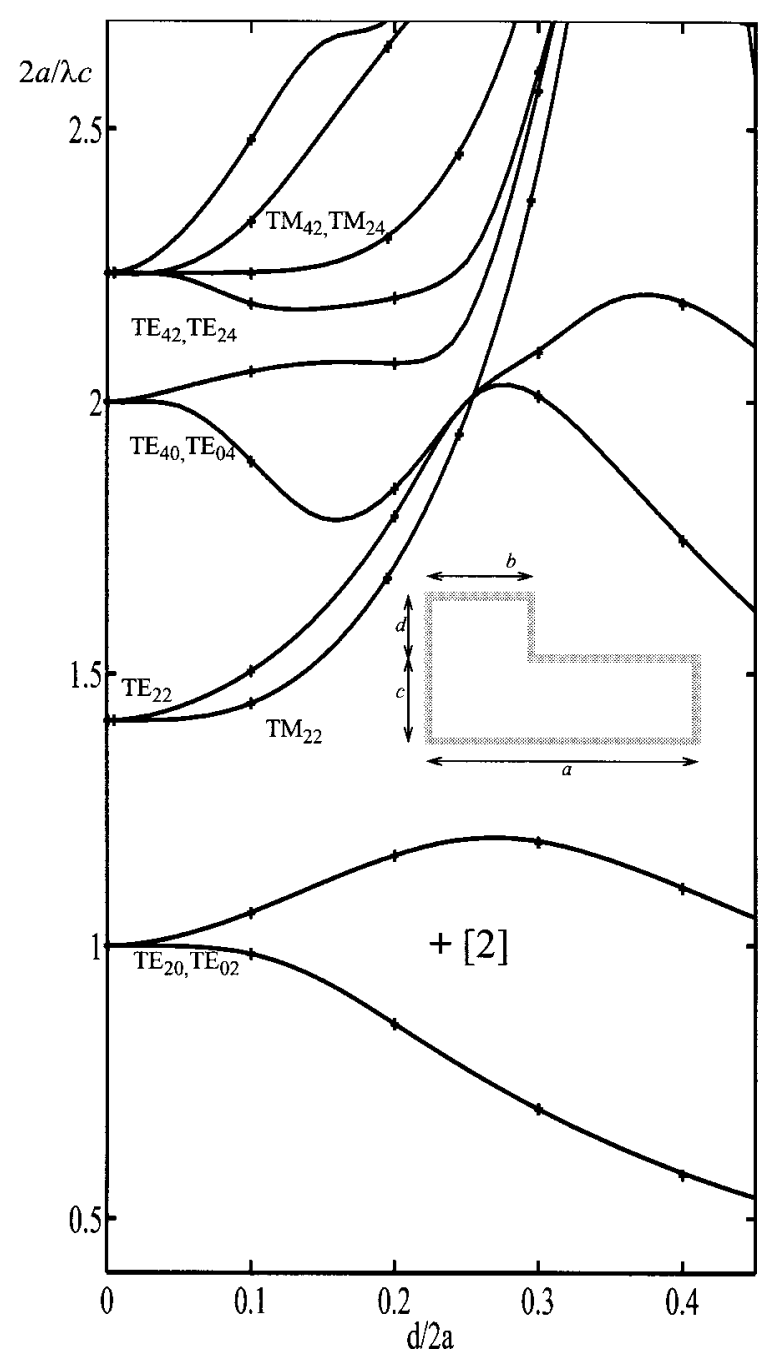

Figure 3. Normalized cut-off frequencies as a function of $d / 2 a$ for a symmetrical L-shaped waveguide with $c=b, d=a-b$. Subscripts $i k, k i$ denote identical cut-off frequencies of modes $i k$ and $k i$ due to symmetry. 


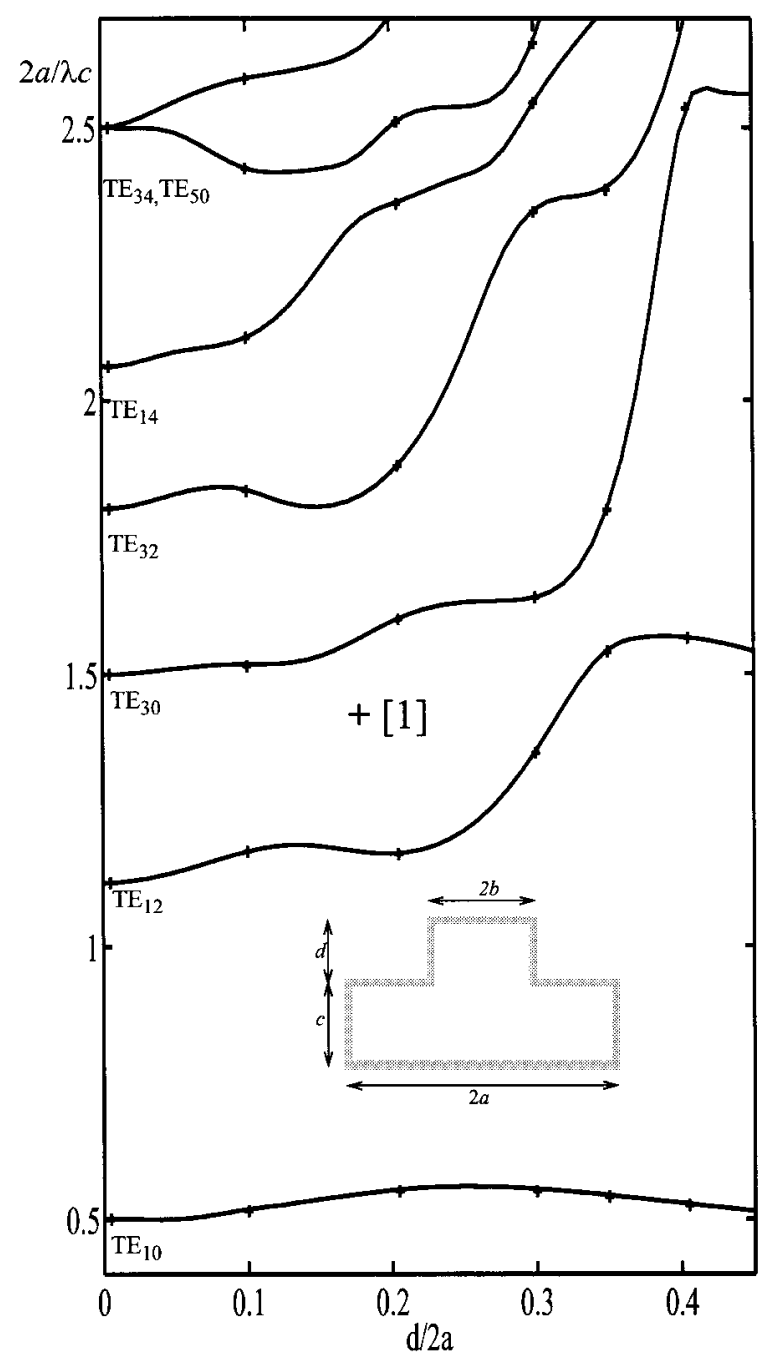

Figure 4. Normalized cut-off frequencies of the TE modes as a function of $d / 2 a$ for a symmetrical $\mathrm{T}$ shaped waveguide with $c=b, d=a-b$, and magnetic wall symmetry.

the additional TE and TM modes of a cross-iris with magnetic-wall symmetry, which completes the mode spectrum of a general cross iris. Again, excellent agreement with [2] is demonstrated.

As a compromise between accuracy and computation time, a $10 \times 10$ matrix was used by Lin [2] for the cross-iris. All results in this paper, however, were generated using only two basis functions, i.e., requiring only a $2 \times 2$ matrix. In most cases, one basis function gives sufficiently accurate results. This efficiency of the method is demonstrated in Table I by the convergence of the first two TE modes with odd-even symmetry. It can be seen that all results converge and agree to within less than $0.1 \%$.

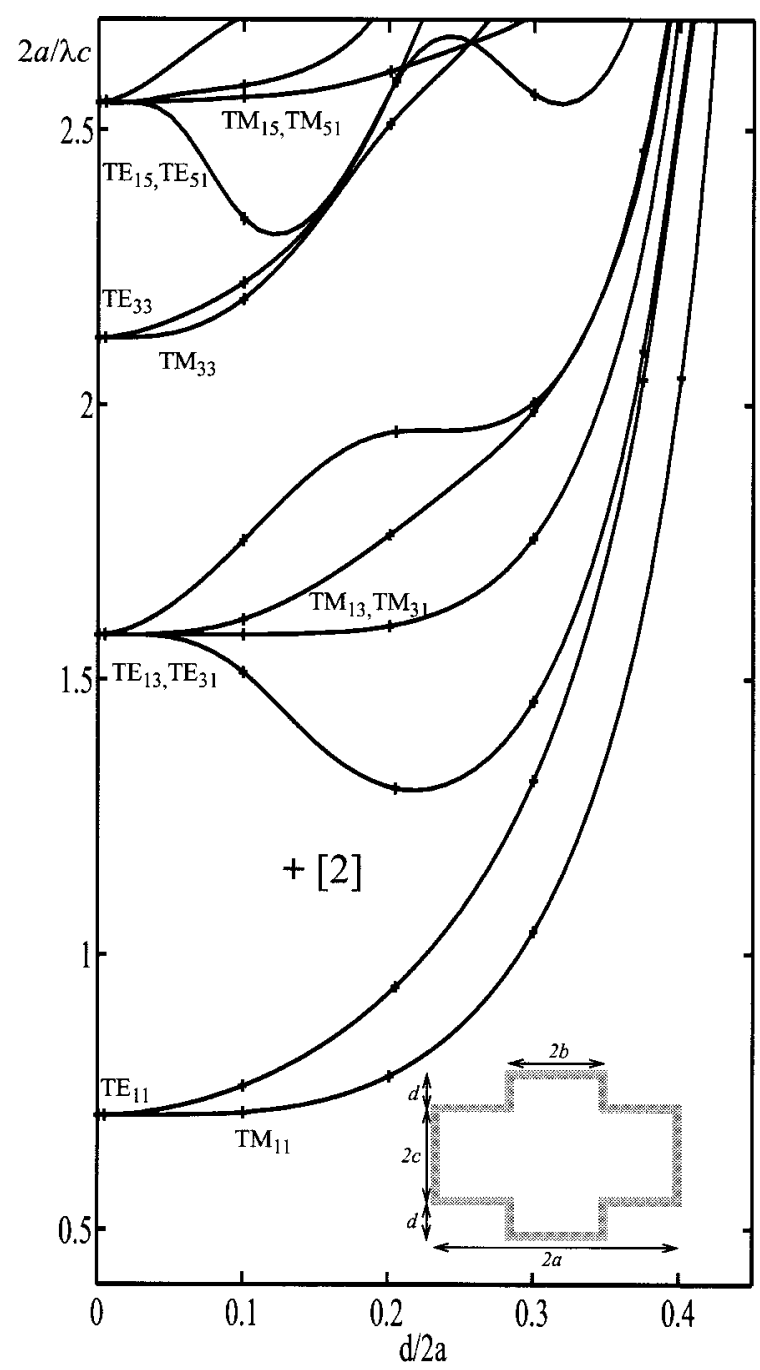

Figure 5. Normalized cut-off frequencies for the additional modes of a symmetrical cross-iris with $c=b$, $d=a-b$ as a function of $d / 2 a$. Subscripts $i k, k i$ denote identical cut-off frequencies of modes $i k$ and $k i$ due to symmetry.

TABLE I. Variation of Normalized Cut-Off Frequencies over Number of Basis Functions

\begin{tabular}{ccc}
\hline $\begin{array}{c}\text { Number of } \\
\text { Basis } \\
\text { Functions }\end{array}$ & $\begin{array}{c}2 a / \lambda_{\mathrm{c}} \text { of } \\
\text { Dominant } \\
\mathrm{TE}_{10} \text { Mode }\end{array}$ & $\begin{array}{c}2 a / \lambda_{\mathrm{c}} \text { of } \\
\mathrm{TE}_{12} \text { Mode }\end{array}$ \\
\hline 1 & 0.52856 & 1.56735 \\
2 & 0.52884 & 1.56764 \\
3 & 0.52887 & 1.56768 \\
4 & 0.52888 & 1.56769 \\
5 & 0.52888 & 1.56769 \\
\hline
\end{tabular}

Cross-iris waveguide with $b / a=0.2, c / a=0.2, d / a=0.8$. 
$2 a / \lambda c$

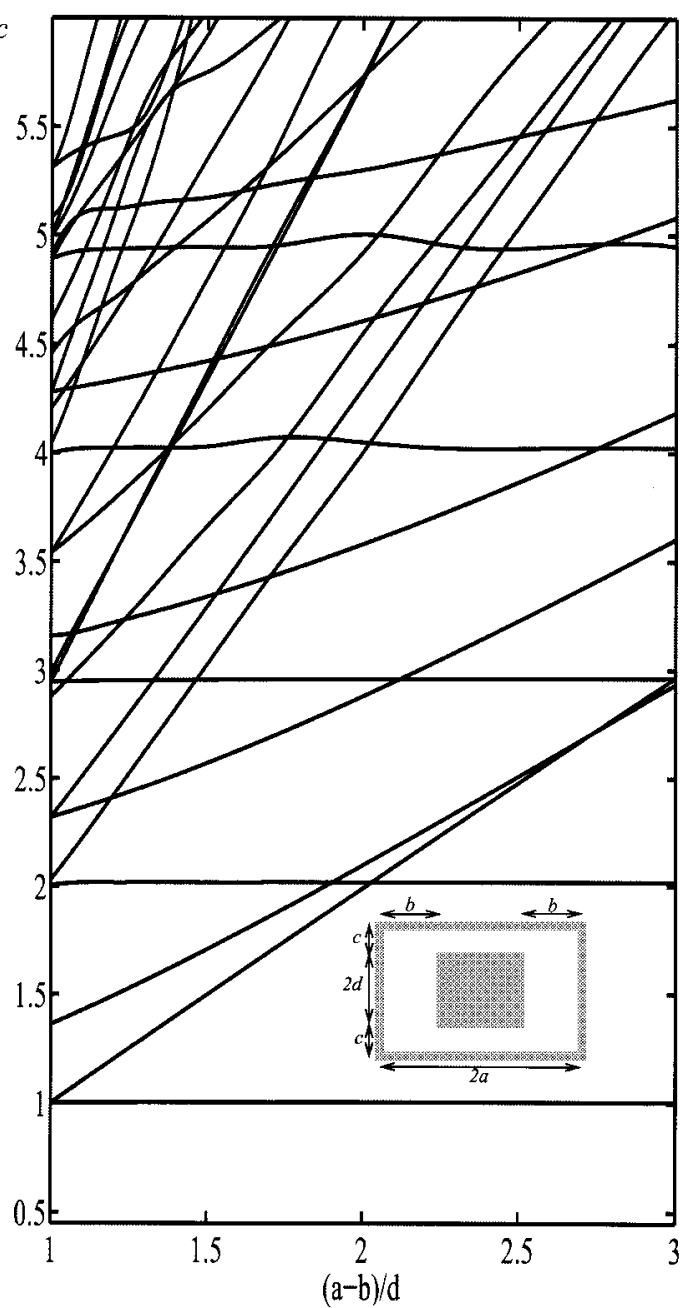

Figure 6. Normalized TE cut-off frequencies as a function of the side ratio $(a-b) / d$ for a rectangular coaxial waveguide with $b / a=c /(c+d)=0.9$.

Next, the technique is applied to the rectangular coaxial waveguide of Fig. 1d. The results for the TE-mode cut-off wave numbers are shown in Figure 6. Identical results were obtained by using standard modal field-matching procedures. However, the results from this integral-equation technique were generated by using only two basis functions. In most cases, one basis function gave accurate results for the wave number, leading to only one scalar to be minimized, whereas in standard modal field matching, the singular value of a $10 \times 10$ matrix was minimized. Similar results are obtained for the TM modes of a rectangular coaxial waveguide (not shown here).

In the example of the rectangular coaxial waveguide of Fig. 1d, Figure 7 compares the con- vergence of the calculated electric field component $E_{x}$ immediately along the interface between the two regions (at $y=d+0.0001 a$; cf. Fig. 1d) by two different techniques. The dotted line represents results from the standard modal fieldmatching technique, using 30 and 300 modes in regions I and II, respectively. Although the approximation of the singularity is clearly visible, the results retain a relatively high ripple, which is typical for the superposition of individual modes. With the same number of modes (300) but with only two edge-conditioned basis functions in the integral-equation technique (dashed line), the ripple is markedly reduced, although the slope at the discontinuity is still far from representing a singular field. The solid line is obtained by the integral-equation technique with two basis functions, but using 2000 modes in the sums of the individual matrix elements. (Note that this does not increase the size of the matrix.) The ripple is reduced significantly, and the slope at the discontinuity represents much more closely that of a field singularity. For this specific case, we have refrained from a direct comparison with the modal field-matching technique, as 2000 modes is quite an unrealistic scenario in mode matching, because of the resulting matrix of exactly this size. The integral-equation technique, however, is capable of including even more modes while maintaining the matrix size, as determined only by the number of basis functions.

Finally, Figure 8 shows the normalized TEmode cutoff frequencies for a ridged circular waveguide of Figure 2. The good agreement with data from a finite-element method [8] demonstrates that the new set of basis functions can also successfully be applied to circular waveguide structures, since the angular dependence is sinusoidal. While two basis functions are usually sufficient for the TM-mode spectrum of such a structure, four to five basis functions are required for the TE mode case with magnetic-wall symmetry in the $x=0$ plane (Fig. 2). This is believed to be due to the distortive influence of the ridges on the fundamental $\mathrm{TE}_{11}$ mode (and related field symmetries) of the empty circular waveguide.

\section{CONCLUSIONS}

A new set of basis functions within an integralequation technique is presented to solve for the 


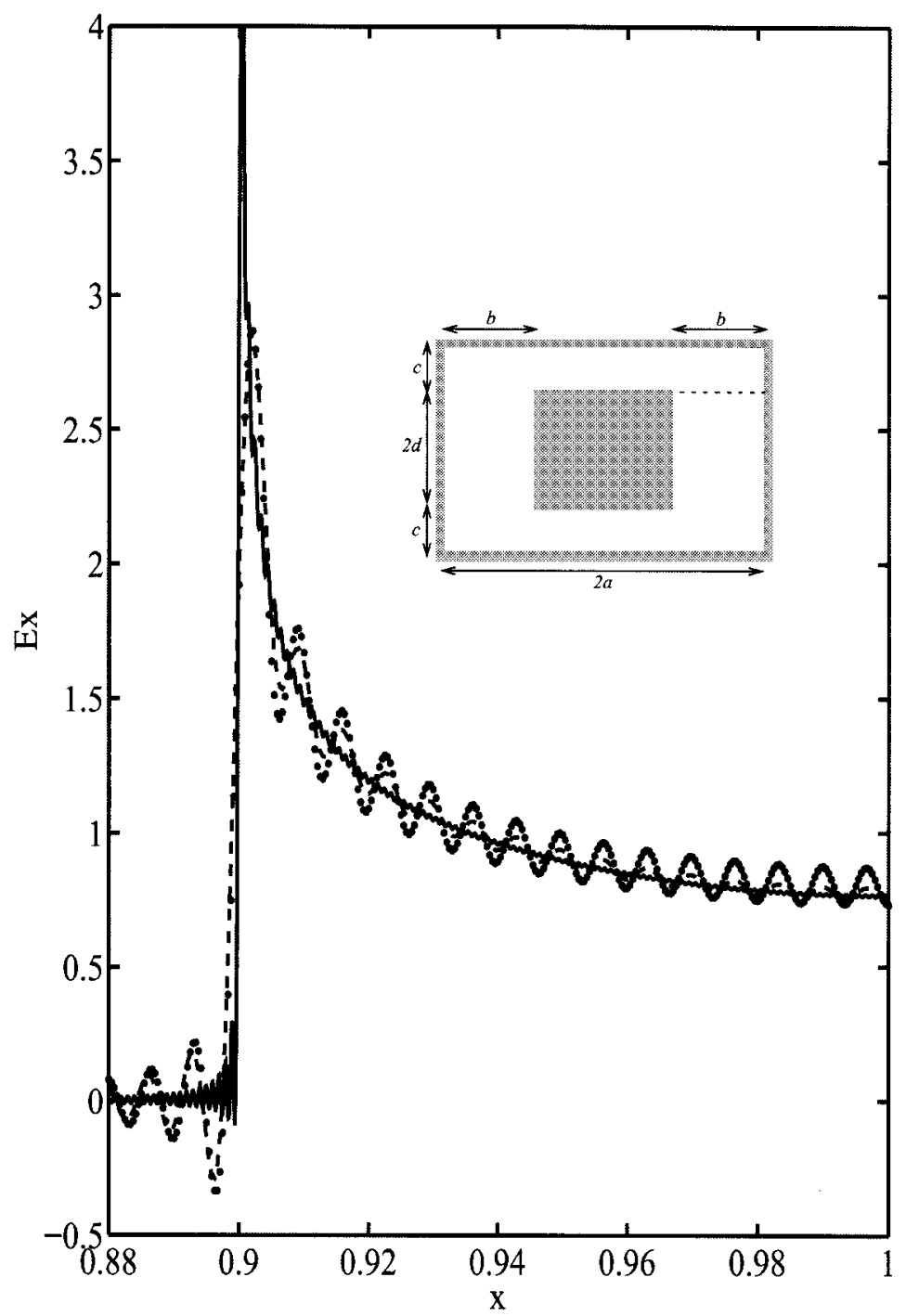

Figure 7. Tangential electric field component $E_{x}$ of a rectangular coaxial waveguide in region II, right next to the interface, at $y=d+0.0001 a$ (cf. Fig. 1d); standard modal field-matching results using 30 and 300 modes in regions I and II (dotted line), integralequation technique using two edge-conditioned basis functions plotted with 300 modes (dashed line) and 2000 modes (solid line). Dimensions: $b / a=0.1, c / a=0.1, d / a=0.9$.

mode spectra and fields of rectangular and circular waveguide structures of nonstandard cross sections. The method is based on directly expanding the tangential electric field at the interface plane in terms of basis functions. These basis functions are chosen so that they, first, satisfy the edge conditions at the metallic corners; second, are analytically Fourier-transformable; and third, include the basic form of the sinusoidal dependence of the waveguide-mode functions. Therefore, the results show rapid convergence in terms of both accuracy and computational speed. Sufficient accuracy is usually obtained by using only one or two basis functions, not only for the cut-off frequencies, but also for the field components in the immediate vicinity of metallic corners. It is 


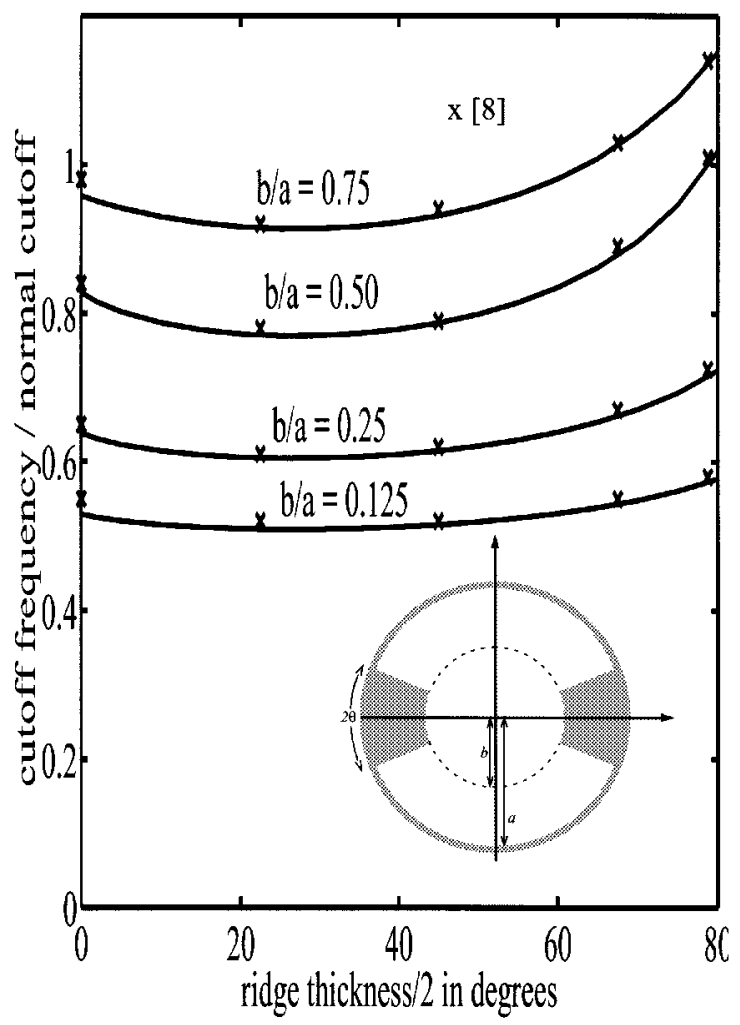

Figure 8. Normalized cut-off frequencies of the TE modes of a ridged circular waveguide as a function of half the ridge angle, $\Theta$, and for different depths of the ridge $b / a$.

obvious that this set of basis functions can be applied to many other structures with $90^{\circ}$ metallic edges and sinusoidal mode distribution.

\section{REFERENCES}

1. R. Ihmels and F. Arndt, "Rigorous Modal S-Matrix Analysis of the Cross-Iris in Rectangular Waveguides," IEEE Microwave Guided Wave Lett., Vol. 2, Oct. 1992, pp. 400-402.

2. F.-L. C. Lin, "Modal Characteristics of Crossed
Rectangular Waveguides," IEEE Trans. Microwave Theory Tech., Vol. MTT-25, Sept. 1977, pp. 756-763.

3. X.-P. Liang, K. A. Zaki, and A. E. Atia, "Dual Mode Coupling by Square Corner Cut in Resonators and Filters," IEEE Trans. Microwave Theory Tech., Vol. 40, Dec. 1992, pp. 2294-2302.

4. B.-T. Lee, J. W. Lee, H. J. Eom, and S.-Y. Shin, "Fourier-Transform Analysis for Rectangular Groove Guide," IEEE Trans. Microwave Theory Tech., Vol. 43, Sept. 1995, pp. 2162-2165.

5. T. Itoh, Ed., Numerical Techniques for Microwave and Millimeter-Wave Passive Structures. John Wiley and Sons, New York, 1989.

6. J. Uher, J. Bornemann, and U. Rosenberg, Waveguide Components for Antenna Feed Systems: Theory and CAD. Artech House, Boston, 1993.

7. I. S. Gradshteyn and I. M. Ryzhik, Tables of Integrals, Series and Products, 5th ed. Academic Press, San Diego, 1994.

8. P. Daly, "Polar Geometry Waveguides by FiniteElement Methods," IEEE Trans. Microwave Theory Tech., Vol. MTT-22, March 1974, pp. 202-209.

9. V. A. Labay and J. Bornemann, "Singular Value Decomposition Improves Accuracy and Reliability of T-Septum Waveguide Field-Matching Analysis," Int J. MIMICAE, Vol. 2, Feb. 1992, pp. 82-89.

10. J. Bornemann and V. A. Labay, "Ridge Waveguide Polarizer with Finite and Stepped-Thickness Septum," IEEE Trans. Microwave Theory Tech., Vol. 43, Aug. 1995, pp. 1782-1787.

11. R. F. Harrington, Field Computation by Moment Methods. Krieger, Malabar, FL, 1987.

12. R. Sorrentino, M. Mongiardo, F. Alessandri, and G. Schiavon, "An Investigation on the Numerical Properties of the Mode-Matching Technique," Int. J. Numer. Model., Vol. 4, Mar. 1991, pp. 19-43.

13. T. Rozzi and M. Mongiardo, "E-Plane Steps in Rectangular Waveguide," IEEE Trans. Microwave Theory Tech., Vol. 39, Aug. 1991, pp. 1279-1288.

14. T. Kitazawa and T. Sugano, "Nonreciprocal Characteristics of Broadside-Coupled Fin Lines with Magnetized Ferrites." 1995 IEEE MTT-S International Microwave Symposium Dig., pp. 729-732.

15. W. H. Press, B. P. Flannery, S. A. Teukolsky, and W. T. Vetterling, Numerical Recipes. The Art of Scientific Computing (Fortran Version), 2nd ed. Cambridge University Press, Cambridge, 1995.

\section{BIOGRAPHIES}

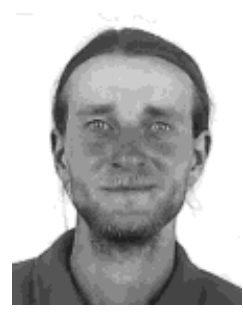

John M. Damaschke was born in 1968 in Hamburg, West Germany. He received his Vordiplom and Dipl.-Ing. in Electrical Engineering from the Technical University Hamburg-Harburg in 1990 and 1994, respectively. During his studies, in 1993, he spent six months in the Department of Electrical Engineering at the University of Manchester, GB, where he worked in power electronics. From 1993 to 1994 he was employed in the Department of Electrical Engineering at the University of Victoria, Canada, where he is currently pursuing the Ph.D. degree. His research interests include numerical modeling in electromagnetics. 


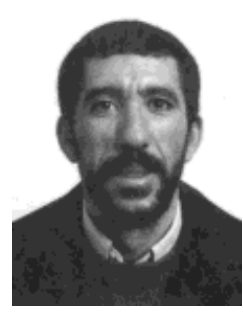

Smain Amari received a D.E.S. in Physics and Electronics from Constantine University (Algeria) in 1985, an M.S. degree in Electrical Engineering in 1989, and a Ph.D. in Physics in 1994, both from Washington University of St. Louis. Since 1994 he has been with the Department of Electrical and Computer Engineering at the University of Victoria, Canada. He is interested in numerical methods in electromagnetics, numerical analysis, applied mathematics, applied physics, and application of quantum field theory in quantum many-particle systems.

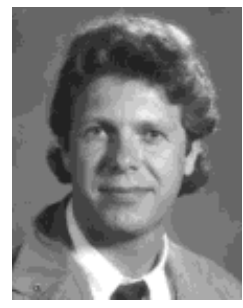

Jens Bornemann received the Dipl.-Ing. and the Dr.-Ing. degrees, both in electrical engineering, from the University of Bremen, Germany, in 1980 and 1984, respectively. From 1980 to 1983 , he was a Research and Teaching Assistant in the Microwave Department at the University of Bremen, where he worked on quasiplanar waveguide configurations and com- puter-aided E-plane filter design. In 1985, after a two-year period as a consulting engineer, he joined the University of Bremen again and was employed at the level of Assistant Professor. Since April 1988, he has been with the University of Victoria, Victoria, B.C., Canada, where he is currently a Professor in the Department of Electrical and Computer Engineering. His research activities include microwave/millimeter-wave components and systems design, and problems of electromagnetic field theory in integrated circuits and radiating structures. He is a coauthor of Waveguide Components for Antenna Feed Systems. Theory and Design (Artech House, 1993) and has authored/coauthored more than 120 technical papers. He is a Registered Professional Engineer in the Province of British Columbia, Canada, and has been a Fellow of the British Columbia Advanced Systems Institute, 1992-1995. Dr. Bornemann was one of the recipients of the A. F. Bulgin Premium of the Institution of Electronic and Radio Engineers in 1983. He is a Senior Member of IEEE and serves on the editorial boards of the IEEE Transactions on Microwave Theory and Techniques and the International Journal of Numerical Modelling. 